EPJ Web of Conferences 92,02082 (2015)

DOI: $10.1051 /$ epjconf/ 20159202082

(C) Owned by the authors, published by EDP Sciences, 2015

\title{
Numerical solution of inviscid and viscous flow around the profile
}

\author{
Martin Slouka, a ${ }^{1,}$ Karel Kozel ${ }^{1}$ and Jaromir Prihoda ${ }^{2}$ \\ ${ }^{1}$ Dept. of Technical Mathematics, Faculty of Mechanical Eng., CTU in Prague, 12135 Prague 1, Czech Rep. \\ ${ }^{2}$ Institute of Thermomechanics, Academy of Science CR, 18200 Prague 8, Czech Rep.
}

\begin{abstract}
This work deals with the $2 \mathrm{D}$ numerical solution of inviscid compressible flow and viscous compressible laminar and turbulent flow around the profile. In a case of turbulent flow algebraic Baldwin-Lomax model is used and compared with Wilcox's $\mathrm{k}-\omega$ model. Calculations are done in GAMM channel computational domain with $10 \%$ DCA profile and in turbine cascade computational domain with $8 \%$ DCA profile. Numerical methods are based on a finite volume solution and compared with experimental measurements for $8 \%$ DCA profile.
\end{abstract}

\section{Introduction}

Mathematical models used in this work are based on the solution of the inviscid compressible flow and viscous compressible turbulent flow over the profile.

Computational domain represents GAMM channel with $10 \%$ DCA profile and blade cascade with $8 \%$ DCA profile. The results show differences between the uses of each numerical model and experimental results.

\section{Governing equations}

The two-dimensional viscous compressible flow is prescribed by the system of Navier-Stokes equations in following conservation form

$$
\mathbf{W}_{\mathrm{t}}+\mathbf{F}_{\mathrm{x}}+\mathbf{G}_{\mathrm{y}}=\mathbf{R}_{\mathrm{x}}+\mathbf{S}_{\mathrm{x}}
$$

where

$$
\begin{aligned}
& \mathbf{W}=(\rho, \rho u, \rho v, e)^{\mathrm{T}}, \\
& \mathbf{F}=\left(\rho u, \rho u^{2}+p, \rho u v,(e+p) u\right)^{\mathrm{T}}, \\
& \mathbf{G}=\left(\rho v, \rho u v, \rho v^{2}+p,(e+p) v\right)^{\mathrm{T}}, \\
& \mathbf{R}=\left(0, \tau_{\mathrm{xx}}, \tau_{\mathrm{xy}}, u \tau_{\mathrm{xx}}+v \tau_{\mathrm{xy}}-q_{\mathrm{x}}\right)^{\mathrm{T}}, \\
& \mathbf{S}=\left(0, \tau_{\mathrm{xy}}, \tau_{\mathrm{yy}}, u \tau_{\mathrm{xy}}+v \tau_{\mathrm{yy}}-q_{\mathrm{y}}\right)^{\mathrm{T}}
\end{aligned}
$$

and

$$
\begin{aligned}
& \tau_{\mathrm{ij}}=\mu\left(\frac{\partial u_{\mathrm{i}}}{\partial x_{\mathrm{j}}}+\frac{\partial u_{\mathrm{j}}}{\partial x_{\mathrm{i}}}\right)-\frac{1}{3} \delta_{\mathrm{ij}} \frac{\partial u_{\mathrm{k}}}{\partial x_{\mathrm{k}}}, \\
& q_{\mathrm{i}}=-\frac{\kappa}{\kappa-1} \frac{\mu}{\operatorname{Pr}} \frac{\partial}{\partial x_{\mathrm{i}}}\left(\frac{p}{\rho}\right)
\end{aligned}
$$

This system is closed by following equation

$$
p=(\kappa-1)\left[e-\frac{1}{2} \rho\left(u^{2}+v^{2}\right)\right] \text {. }
$$

In the equations above $\rho$ denotes density, $(u, v)$ are components of local velocity in $x$ and $y$ direction respectively, $e$ denotes total energy per unit volume, $p$ is pressure given by previous equation of state, $\tau_{\mathrm{ij}}$ is shear stress, $q_{\mathrm{i}}$ represents heat flux, $\mu$ represents dynamical viscosity.

Following constants were used in previous equations: $\kappa$ is isentropic exponent equalled to $1.4, \mathrm{Pr}$ is Prandtl's number equalled to 0.7 .

Viscous compressible turbulent flow represents system of Reynolds averaged Navier-Stokes equations (RANS) which is formally the same as (1) with enclosed used model of turbulence which is algebraic BaldwinLomax or two-equation Wilcox's k- $\omega$ model in this work.

Inviscid flow represented by the system of Euler equations is simply reached after neglecting of viscosity in viscous fluxes on the right hand side in the system (1).

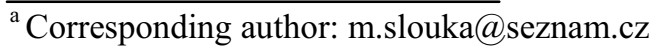




\section{Turbulence models}

\subsection{Baldwin-Lomax model}

Algebraic turbulent models are based on the model proposed for boundary layer flow by Cebeci and Smith. Baldwin-Lomax model is the modification of basic Cebeci and Smith model. The boundary layer is divided into two regions, inner region (close to the wall) and outer region. Turbulent viscosity in the inner part is given by

$$
\mu_{\mathrm{ti}}=\rho F_{\mathrm{d}}^{2} \kappa^{2} y^{2}|\Omega|
$$

where vorticity is in $2 \mathrm{D}$ case defined as

$$
\Omega=\frac{\partial u}{\partial y}-\frac{\partial v}{\partial x}
$$

van Driest function given as follow

$$
F_{\mathrm{d}}=1-\exp \left(-\frac{1}{A} \frac{u_{\tau} y}{v_{\mathrm{w}}}\right)
$$

and friction velocity by formula

$$
u_{\tau}=\sqrt{\frac{\tau_{\mathrm{w}}}{\rho}}=\left(v \frac{\partial u}{\partial y}\right)_{\mathrm{w}}^{1 / 2}
$$

Turbulent viscosity in outer region is given by

$$
\mu_{\mathrm{to}}=\alpha C_{\mathrm{cp}} F_{\mathrm{w}} F_{\mathrm{k}}
$$

Function $F_{\mathrm{w}}$ is determined by the relation

$$
F_{\mathrm{w}}=y_{\max } F_{\max }
$$

where $F_{\mathrm{w}}$ is the maximum of the function bellow

$$
F=y|\Omega| F_{\mathrm{d}}
$$

and $y_{\max }$ is the distance from the wall in which $F\left(y_{\max }\right)=F_{\text {max }}$ holds and

$$
F_{\mathrm{k}}=\left[1+5.5\left(C_{\mathrm{KL}} \frac{y}{y_{\max }}\right)^{6}\right]^{-1}
$$

In Baldwin-Lomax turbulent model following constants are used: $\kappa=0.4, A=26, \alpha=0.0168, C_{\mathrm{cp}}=1.6$ and $C_{\mathrm{KL}}=0.3$.

\section{$3.2 \mathrm{k}-\omega$ model}

The two-equation model is given by transport equations for two characteristic scales of turbulent motion, in this case turbulent energy $k$ and specific dissipation rate $\omega$. Then turbulent viscosity is defined as

$$
\mu_{\mathrm{t}}=\rho \frac{k}{\omega} .
$$

The Wilcox's k- $\omega$ model was used as follow

$$
\begin{aligned}
& \frac{\partial(\rho k)}{\partial t}+\frac{\partial\left(\rho u_{\mathrm{j}} k\right)}{\partial x_{\mathrm{j}}}=P_{\mathrm{k}}+\frac{\partial}{\partial x_{\mathrm{j}}}\left[\left(\mu+\sigma^{*} \mu_{\mathrm{t}}\right) \frac{\partial k}{\partial x_{\mathrm{j}}}\right]-\beta^{*} \rho k \omega \\
& \frac{\partial(\rho \omega)}{\partial t}+\frac{\partial\left(\rho u_{\mathrm{j}} \omega\right)}{\partial x_{\mathrm{j}}}=\gamma \frac{\omega}{k} P_{\mathrm{k}}+\frac{\partial}{\partial x_{\mathrm{j}}}\left[\left(\mu+\sigma \mu_{\mathrm{t}}\right) \frac{\partial \omega}{\partial x_{\mathrm{j}}}\right]-\beta \rho k \omega^{2}
\end{aligned}
$$

where

$$
P_{\mathrm{k}}=\tau_{\mathrm{ij}} \frac{\partial u_{\mathrm{i}}}{\partial x_{\mathrm{j}}}
$$

represents production of turbulent energy.

Following model coefficients are used: $\alpha=5 / 9, \beta=$ $3 / 40, \beta^{*}=9 / 100$ and $\sigma=\sigma^{*}=1 / 2$.

\section{Numerical method}

\subsection{Numerical scheme}

Finite volume method was applied on cell centred nonorthogonal structured grid with quadrilateral cells.

For numerical solutions Lax-Wendroff scheme in McCormack modification was used with predictor step

$$
\mathbf{W}_{\mathrm{i}, \mathrm{j}}^{\mathrm{n}+1 / 2}=\mathbf{W}_{\mathrm{i}, \mathrm{j}}^{\mathrm{n}}-\Delta t \operatorname{Res} \mathbf{W}_{\mathrm{i}, \mathrm{j}}^{\mathrm{n}}
$$

and corrector step as follow

$$
\mathbf{W}_{\mathrm{i}, \mathrm{j}}^{\mathrm{n}+1}=\frac{1}{2}\left(\mathbf{W}_{\mathrm{i}, \mathrm{j}}^{\mathrm{n}}+\mathbf{W}_{\mathrm{i}, \mathrm{j}}^{\mathrm{n}+1 / 2}-\Delta t \operatorname{Res} \mathbf{W}_{\mathrm{i}, \mathrm{j}}^{\mathrm{n}+1 / 2}\right) .
$$

The Jameson's artificial dissipation model was applied to damp the oscillations.

$$
\begin{gathered}
\tilde{\mathbf{W}}_{\mathrm{i}, \mathrm{j}}^{\mathrm{n}+1}=\mathbf{W}_{\mathrm{i}, \mathrm{j}}^{\mathrm{n}+1}+A D\left(\mathbf{W}_{\mathrm{i}, \mathrm{j}}^{\mathrm{n}}\right), \\
A D\left(\mathbf{W}_{\mathrm{i}, \mathrm{j}}^{\mathrm{n}}\right)=C_{1} \psi_{1}\left(\mathbf{W}_{\mathrm{i}+1, \mathrm{j}}^{\mathrm{n}}-2 \mathbf{W}_{\mathrm{i}, \mathrm{j}}^{\mathrm{n}}+\mathbf{W}_{\mathrm{i}-1, \mathrm{j}}^{\mathrm{n}}\right) \\
+C_{2} \psi_{2}\left(\mathbf{W}_{\mathrm{i}, \mathrm{j}-1}^{\mathrm{n}}-2 \mathbf{W}_{\mathrm{i}, \mathrm{j}}^{\mathrm{n}}+\mathbf{W}_{\mathrm{i}, \mathrm{j}-1}^{\mathrm{n}}\right), \\
\psi_{1}=\frac{\left|p_{\mathrm{i}+1, \mathrm{j}}^{\mathrm{n}}-2 p_{\mathrm{i}, \mathrm{j}}^{\mathrm{n}}+p_{\mathrm{i}-1, \mathrm{j}}^{\mathrm{n}}\right|}{\left|p_{\mathrm{i}+1, \mathrm{j}}^{\mathrm{n}}\right|+\left|p_{\mathrm{i}, \mathrm{j}}^{\mathrm{n}}\right|+\left|p_{\mathrm{i}-1, \mathrm{j}}^{\mathrm{n}}\right|} \\
\psi_{2}=\frac{\left|p_{\mathrm{i}, \mathrm{j}+1}^{\mathrm{n}}-2 p_{\mathrm{i}, \mathrm{j}}^{\mathrm{n}}+p_{\mathrm{i}, \mathrm{j}-1}^{\mathrm{n}}\right|}{\left|p_{\mathrm{i}, \mathrm{j}+1}^{\mathrm{n}}\right|+\left|p_{\mathrm{i}, \mathrm{j}}^{\mathrm{n}}\right|+\left|p_{\mathrm{i}, \mathrm{j}-1}^{\mathrm{n}}\right|} .
\end{gathered}
$$




\subsection{Boundary conditions}

Inlet boundary conditions were realized for inviscid flow as follow: inlet velocity $M a_{\infty}$ together with angle of attack $\alpha$, density $\rho_{\infty}$ and total energy per volume $e_{\infty}$ were set; pressure $p_{\infty}$ was extrapolated from the flow field. In a case of viscous flow the inlet pressure $p_{\infty}$ was set.

Outlet boundary conditions were the same for the both type of flow. Outlet pressure was set and other variables extrapolated from the flow field.

Solid wall condition was realized by the adding of virtual cells. In a case of inviscid flow velocity components were prescribed so that sum of velocity vectors equals zero in its tangential component. In a case of viscous flow velocity components were prescribed so that sum of velocity vectors equals zero on the wall.

\section{Numerical results and discussion}

Mach number isolines are shown in all pictures together with inlet flow parameters below where $M a_{\infty}$ corresponds to the inlet Mach number and $\alpha$ is attack angle of inlet flow. Numerical results were validated for different setting of artificial dissipation to damp the oscillations and in a case of viscous model the influence on the physical viscosity was checked.

First three pictures correspond with results of calculation of transonic inviscid and viscous turbulent flow in GAMM channel. This configuration was set as a reference for testing of behaviour of both models.

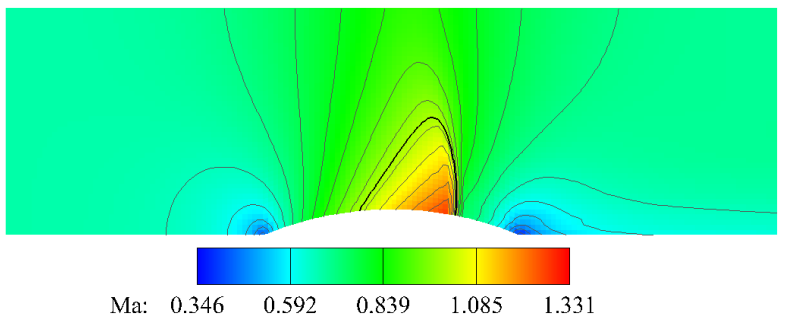

Fig. 1. GAMM channel, inviscid flow, $\mathrm{Ma}_{\infty}=0.675, \alpha=0.0^{\circ}$

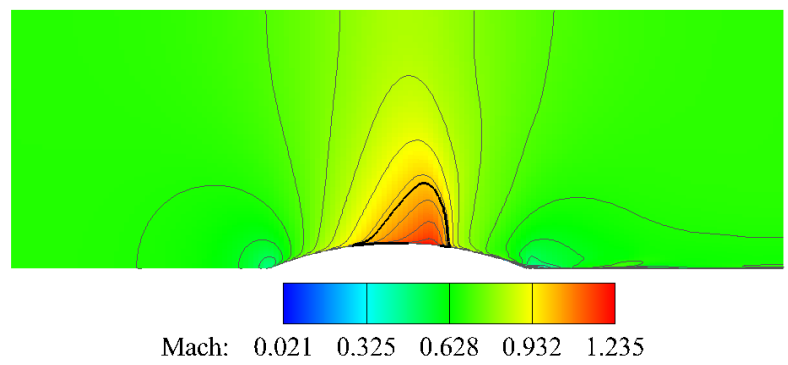

Fig. 2. GAMM channel, viscous turbulent flow, $\mathrm{Ma}_{\infty}=0.675$, $\alpha=0.0^{\circ}, \operatorname{Re}=10^{7}$, Baldwin-Lomax model

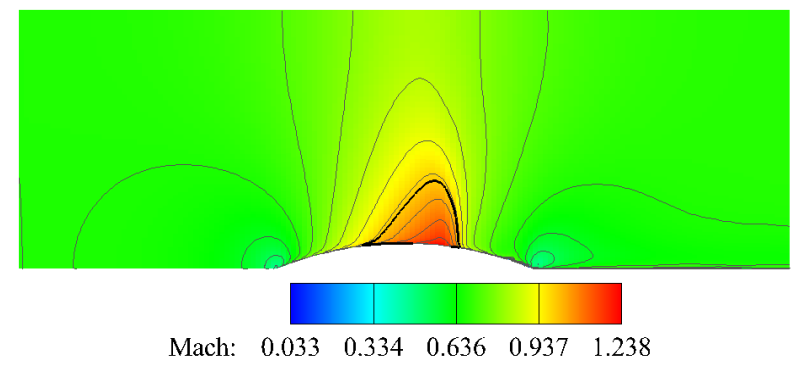

Fig. 3. GAMM channel, viscous turbulent flow, $\mathrm{Ma}_{\infty}=0.675$, $\alpha=0.0^{\circ}, \mathrm{Re}=10^{7}, \mathrm{k}-\omega$ model

Next set of pictures shows comparison between the used inviscid and viscous turbulent flow models and experimental data of measurements of transonic flow through DCA cascade which were done by Institute of Thermomechanics AS CR in Prague and published in [1] and [2]. Here are shown numerical results for two different inlet Mach numbers $M a_{\infty}=0.88$ and $M a_{\infty}=0.98$ and compared with experimental results for inlet Mach numbers $M a_{\infty}=0.832$ and $M a_{\infty}=0.863$ respectively.

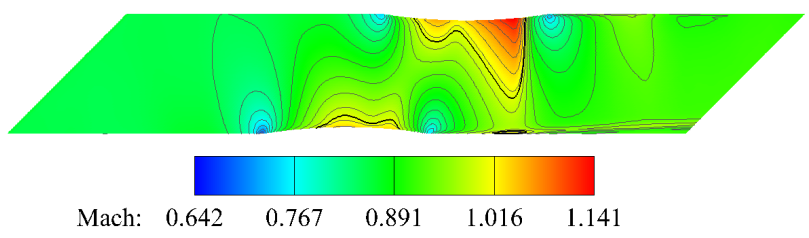

Fig. 4. DCA cascade, inviscid flow, $\mathrm{Ma}_{\infty}=0.88, \alpha=0.6^{\circ}$

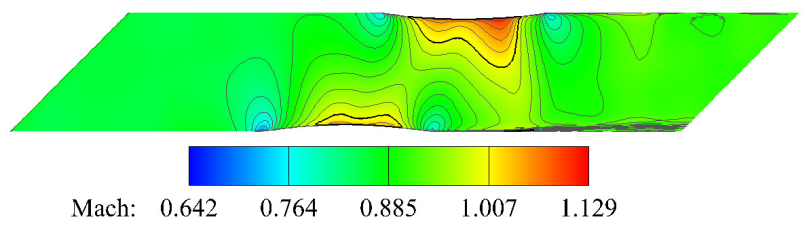

Fig. 5. DCA cascade, viscous turbulent flow, $\mathrm{Ma}_{\infty}=0.88$, $\alpha=0.6^{\circ}, \operatorname{Re}=10^{7}$, Baldwin-Lomax model

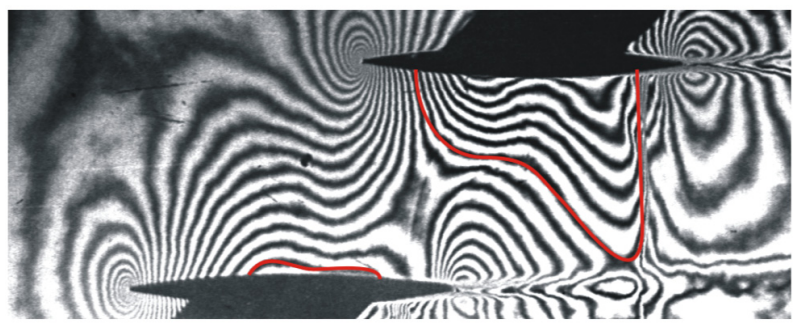

Fig. 6. Experimental data, $\mathrm{Ma}_{\infty}=0.832$ 


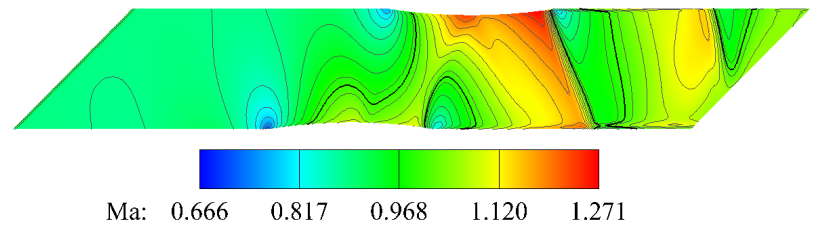

Fig. 7. DCA cascade, inviscid flow, $\mathrm{Ma}_{\infty}=0.98, \alpha=-3.0^{\circ}$

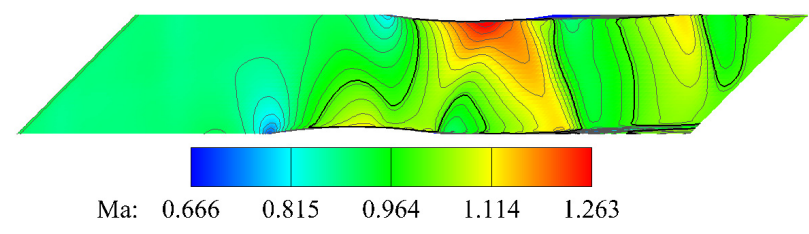

Fig. 8. DCA cascade, viscous turbulent flow, $\mathrm{Ma}_{\infty}=0.98$, $\alpha=-3.0^{\circ}, \operatorname{Re}=10^{7}, \mathrm{k}-\omega$ model

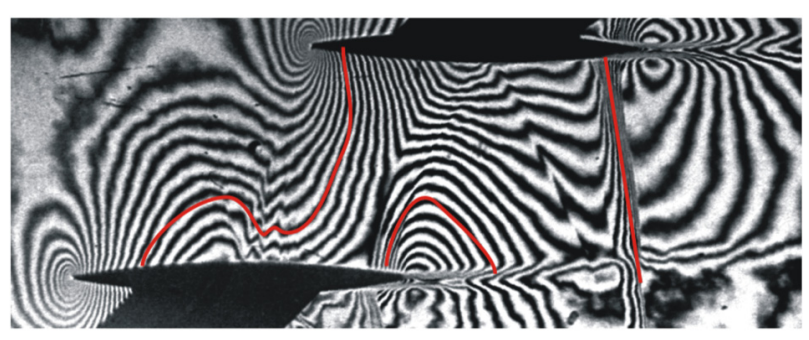

Fig. 9. Experimental data, $\mathrm{Ma}_{\infty}=0.863$

At all pictures the differences between the inviscid and viscous models are shown. In a case of DCA cascade for inlet Mach numbers $M a_{\infty}=0.88$ and angle of attack $\alpha=0.6^{\circ}$ we can also see the differences between the result of viscous model with enclosed Baldwin-Lomax model of turbulence and the experiment. On the other hand in a case of DCA cascade for inlet Mach numbers $M a_{\infty}=0.98$ and angle of attack $\alpha=-3.0^{\circ}$ we can see quite good correlation between the result of viscous model with enclosed $\mathrm{k}-\omega$ model of turbulence and the experiment. This fact of better correlation can also be expected for inlet Mach number $M a_{\infty}=0.88$ and angle of attack $\alpha=0.6^{\circ}$ and $\mathrm{k}-\omega$ model of turbulence.

It is shown that in all cases of flow through DCA cascade inlet Mach number is set slightly bigger than in experiment, also with changing of angle of attack around the zero position we can modify the size and location of sonic isolines on the profiles.

\section{Conclusions}

The developed software was successfully tested for the calculations of inviscid and viscous turbulent flow in GAMM channel and in DCA blade cascade with small region of separation. In a case of DCA blade cascade possibilities of different numerical approaches were shown and compared with experimental data. Achieved results are comparable with other authors [7], [8].

\section{Acknowledgements}

The research has been financed in part by grants P 101/10/1329 and P 101/12/1271 and by the Education for Competitiveness Operation Programme.

\section{References}

1. R. Dvorak, Transonic Flows (Academia, Prague, 1986)

2. R. Dvorak, K. Kozel, Mathematical modelling in aerodynamics (CTU in Prague, Prague, 1996)

3. J. Fort, K. Kozel, P. Louda, J. Fürst, Num. solution of flow problems III (CTU in Prague, Prague 2004)

4. M. Slouka, K. Kozel, Topical Prob. of Fluid Mech. 2014, 127-130 (2014)

5. M. Slouka, K. Kozel, Topical Prob. of Fluid Mech. 2013, 57-60, (2013)

6. M. Slouka, K. Kozel, Fluid Dynamics 2013, 29-30, (2013)

7. J. Trefilik, K. Kozel, Recent Adv. in Fluid Mech. and Heat \& Mass Transfer, 193-196, WSEAS, (2011)

8. J. Trefilik, J. Huml, K. Kozel, J. Prihoda, Czech Japanese Sem. in Applied Math. 2010, 94-107, (2010) 